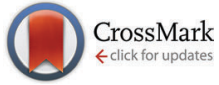

Cite this: Phys. Chem. Chem. Phys., 2014, 16, 26539

Received 16th May 2014, Accepted 15th July 2014 DOI: $10.1039 / c 4 c p 02147 a$

www.rsc.org/pccp

\title{
DFT studies of oxygen dissociation on the 116-atom platinum truncated octahedron particle $\dagger$
}

\author{
Paul C. Jennings, $\ddagger^{\mathrm{a}}$ Hristiyan A. Aleksandrov, ${ }^{\mathrm{bc}}$ Konstantin M. Neyman ${ }^{\text {bd }}$ and \\ Roy L. Johnston*e
}

\begin{abstract}
Density functional theory calculations are performed to investigate oxygen dissociation on 116-atom truncated octahedron platinum particles. This work builds on results presented previously [Jennings et al., Nanoscale, 2014, 6, 1153], where it was shown that shell flexibility played an important role in facilitating fast oxygen dissociation. In this study, through investigation of the larger particle size, it is shown that oxygen dissociation on the (111) facet of pure platinum species is still aided by shell flexibility at larger sizes. Only the hollow sites close to the edges of the (111) facet mediate oxygen dissociation; oxygen is bound too weakly at other hollow sites for dissociation to occur. Further studies are performed on the (100) facet, which is larger for the $\mathrm{Pt}_{116}$ particle than for either the $\mathrm{Pt}_{38}$ or $\mathrm{Pt}_{79}$ ones. Much higher dissociation barriers are found on the (100) facet compared to the (111) facet, where the bridge sites are favourable for oxygen dissociation.
\end{abstract}

\section{Introduction}

The interest in platinum (Pt) systems is due in part to the relevance of Pt based catalysts in many industrial applications, such as catalysing the oxygen reduction reaction (ORR) in hydrogen fuel cells. ${ }^{1-4}$ For low temperature fuel cells, a common problem associated with the use of a Pt electrocatalyst is the strong Pt-O binding, which aids oxygen dissociation, but is responsible for reducing the rate of the ORR..$^{5-7}$ Modelling of the ORR is a very active area of research, with emphasis on computing the overall mechanism, ${ }^{8-12}$ as well as chemisorption studies of specific intermediates within the reaction. ${ }^{13-15}$

Oxygen chemisorption studies have been performed on a number of extended Pt surfaces, ${ }^{16-18}$ although there is also significant interest in the study of both free ${ }^{19-21}$ and supported particle systems. ${ }^{22,23}$ Nanoparticle models can offer a greater number of symmetry inequivalent sites at which to bind oxygen and in the size regime where "each atom counts", ${ }^{24}$ properties

\footnotetext{
${ }^{a}$ School of Chemical Engineering, University of Birmingham, Birmingham, UK

${ }^{b}$ Departament de Química Física \& IQTCUB, Universitat de Barcelona, Spain

${ }^{c}$ Faculty of Chemistry and Pharmacy, University of Sofia, Sofia, Bulgaria

${ }^{d}$ Institució Catalana de Recerca i Estudis Avançats (ICREA), Barcelona, Spain

${ }^{e}$ School of Chemistry, University of Birmingham, Birmingham, UK.

E-mail: r.l.johnston@bham.ac.uk; Fax: +44 (0)121414 4403;

Tel: +44 (0)121 4147477

$\dagger$ Electronic supplementary information (ESI) available: Showing images of all initial, transition and final states for the sites investigated. See DOI: 10.1039/ c4cp02147a

\$ Present address: Department of Energy Conversion and Storage, Technical University of Denmark, Roskilde, Denmark.
}

are often dominated by quantum effects. For these structures, the under-coordinated surface atoms of the particles provide a diverse range of electronic environments at which to bind $0 .^{25}$ However, it should be noted that it is also possible to use particle models that are scalable with size. ${ }^{26-28}$ In the work presented here, focus is placed on investigating $\mathrm{O}_{2}$ dissociation on Pt particles. The behaviour of $\mathrm{O}_{2}$ dissociation on Pt surfaces has been studied extensively. Whilst this has predominantly focused on bulk systems, ${ }^{29-36}$ some studies have been performed on small particles. ${ }^{37-40}$

In work published previously, we showed that when Pt is alloyed with titanium (Ti) to produce core-shell Ti@Pt moieties, changes in the electronic structure of the nanoparticle result in weaker Pt-O binding, through filling of the Pt d-band; ${ }^{41,42}$ where similar trends have been found for a number of other Pt based alloy systems. ${ }^{43-45}$ In later studies, it was found that whilst the strong core-shell interactions could result in a favourable reduction of $\mathrm{Pt}-\mathrm{O}$ binding energies, these strong $\mathrm{Pt}-\mathrm{M}$ interactions also inhibited oxygen dissociation. Shell flexibility plays an important role in facilitating fast oxygen dissociation, where on pure Pt particles the outermost shell can distort in such a way as to reduce the $\mathrm{O}_{2}$ dissociation barrier from $0.4 \mathrm{eV}$ on the (111) bulk slab, to $<0.1 \mathrm{eV}$ on the (111) facet of the particle. ${ }^{46}$

The previous studies on oxygen dissociation were performed on 38- and 79 -atom particles. ${ }^{46}$ It is noted that other studies found significant variation in adsorption energies of $\mathrm{O}$ on small Pt particles, when nearby facets were modified; ${ }^{47}$ although, this appears less pronounced for particle sizes $>80$ atoms. Therefore, size effects potentially play an important role in $\mathrm{O}$ binding as well as flexibility of the Pt shell for the small particle sizes 
investigated previously. In the work presented here, $\mathrm{O}_{2}$ dissociation studies are performed on the larger truncated octahedron (TO) $\mathrm{Pt}_{116}$ nanoparticles, which should be in the range that scales with size. It allows the investigation of, not only the size dependance of (111) facet flexibility, but also perform a more detailed study of the (100) facet. Although in the previous studies the (100) facet exhibited far higher dissociation barriers, the $\mathrm{Pt}_{116}(100)$ facet is larger and allows for additional $\mathrm{O}_{2}$ adsorption sites to be investigated.

\section{Methodology}

Calculations are performed using the Vienna Ab-initio Simulation Package (VASP) 5.2. ${ }^{48-51}$ For all atom types, the valence electrons are treated explicitly, while the ionic cores are represented by the projected augmented wave (PAW) method. ${ }^{52,53}$ Electronic exchange and correlation are described using the generalised gradient approximation (GGA) using the Perdew-Wang 91 (PW91) formula. ${ }^{54,55}$ The Methfessel-Paxton smearing method is used with a width of $0.1 \mathrm{eV}$ (extrapolated for computing final energies at zero smearing) and an energy cutoff of $415.0 \mathrm{eV}$ has been employed. All particles are placed in the centre of large enough supercells to ensure sufficient separation of $10 \AA$ between periodic images. The $\Gamma$ point is used to sample the Brillouin Zone. Atoms are then relaxed according to the calculated atomic forces, with convergence criteria for energies and forces required to be better than $1.0 \times 10^{-4} \mathrm{eV}$ per atom and

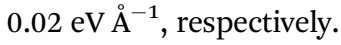

Dissociation pathways are investigated using the Transition State Tools implementation of the VASP code, by first generating an approximate pathway using the Nudged Elastic Band (NEB) method ${ }^{56}$ with further refinements achieved using the Dimer method. ${ }^{57}$ Charges are calculated using the Bader method. ${ }^{58,59}$

In order to assess the (atomic or molecular) oxygen adsorption strength to the Pt surface, the binding energy $\left(E_{\mathrm{b}}\right)$ is calculated, as defined in eqn (1).

$$
E_{\mathrm{b}}=E_{\mathrm{AB}}-\left(E_{\mathrm{A}}+E_{\mathrm{B}}\right)
$$

$E_{\mathrm{AB}}$ is the energy of the particle with oxygen adsorbed, $E_{\mathrm{A}}$ is the energy of the relaxed particle and $E_{\mathrm{B}}$ is the energy of the free oxygen atom or molecule, negative $E_{\mathrm{b}}$ values imply favourable binding. The interaction energy $\left(E_{\text {int }}\right)$ is defined in eqn (2).

$$
E_{\text {int }}=E_{\mathrm{AB}}-\left(E_{\mathrm{A}}^{*}+E_{\mathrm{B}}^{*}\right)
$$

The single point energies of species distorted upon adsorption are denoted by *, again negative values imply favourable interactions. Distortion energies can be calculated using eqn (3).

$$
\Delta E_{\mathrm{A}}=E_{\mathrm{A}}-E_{\mathrm{A}}^{*}
$$

Although specific distortions, brought about by adsorption of oxygen, are discussed in detail, as a more general measure root mean squared displacements (RMSD) of atoms are calculated as defined in eqn (4).

$$
\operatorname{RMSD}=\sqrt{\frac{1}{N} \sum_{i}^{N}\left(m_{i}-m_{i}^{*}\right)}
$$

$N$ is the total number of atoms, $m_{i}$ is the position of a given atom for the relaxed bare $\mathrm{Pt}_{116}$ and $m_{i}^{*}$ is the position of the same atom in the particle following oxygen adsorption. Prior to applying this equation, rotation and translation operators are performed to align each particle, thereby minimising the RMSD. In this study, RMSD is calculated for Pt atoms to which $\mathrm{O}_{2}$ is bound, as well as the nearest neighbour metal atoms.

\section{Results and discussion}

\subsection{Oxygen adsorption}

In the previous studies, the $\mathrm{Pt}_{79}$ TO model showed similar trends to the $\mathrm{Pt}_{38}$ TO, although further site dependent trends were observed for the 79-atom particle that were not present on the limited (111) facet of the 38-atom particle. Therefore, in this study, one further particle size is considered for the pure Pt system. On the surface of the 116 -atom TO model there are 15 symmetry inequivalent sites which are depicted in Fig. 1. The results of atomic and molecular oxygen adsorption on $\mathrm{Pt}_{116}$ are presented in Table 1.

Atomic oxygen is preferentially adsorbed at site 9, the edgebridge position between the (111) and (100) facets, with a binding energy of $-5.02 \mathrm{eV}$. This is in agreement with the studies on the $\mathrm{Pt}_{79}$ model. Whilst the atomic oxygen preferentially binds to the hcp hollow site (more stable by $\geq 0.09 \mathrm{eV}$ than the edge-bridge sites) on the 38-atom moiety, favourable binding is observed to the edge-bridge position between two (111) facets (strengthened by $\geq 0.06 \mathrm{eV}$ compared to hollow sites). This shows that there is competition between atomic oxygen adsorption on the edge-bridge and hollow sites on the 38- and 79-atom particles. However, on the 116-atom particle, the edge-bridge site between the (111) and (100) facets are found to be $\geq 0.23 \mathrm{eV}$ more stable

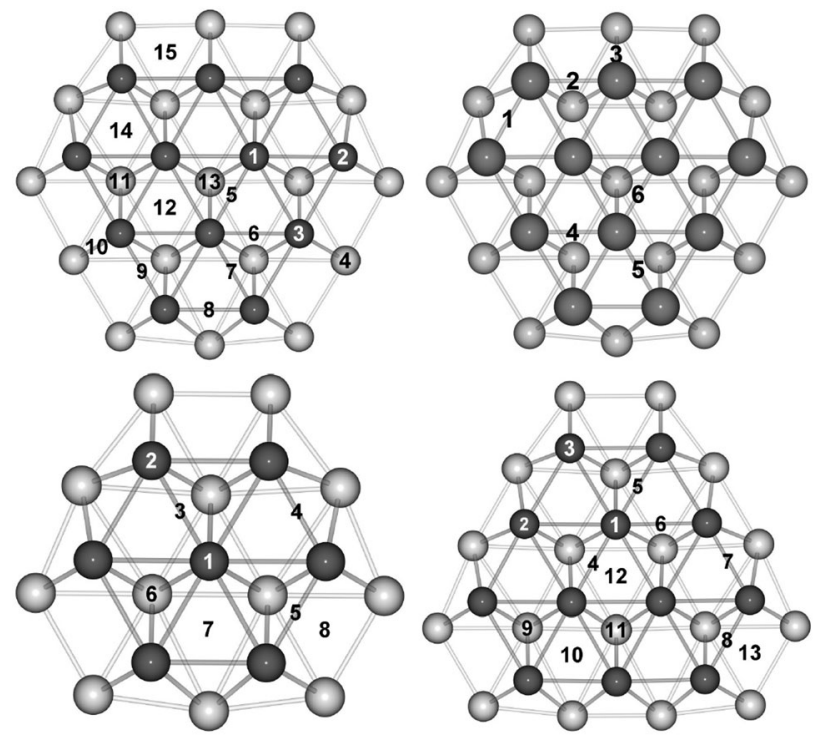

Fig. 1 Top view of the surface (dark grey) and subsurface (light grey) metal atoms at a (111) facet showing symmetry inequivalent adsorption sites [top left] and bond numbering [top right] for $\mathrm{TO}^{\mathrm{Pt}_{116}}$. For reference, the numbering used for $\mathrm{Pt}_{38}$ [bottom left] and $\mathrm{Pt}_{79}$ [bottom right], is also shown. 
Table 1 Calculated binding energies $\left(E_{\mathrm{b}}\right)$ for atomic and molecular oxygen on all sites of the $\mathrm{Pt}_{116}$ model, shown in Fig. 1. Values not presented are the result of oxygen migration to other sites during relaxation, as labelled. All energies are given in $\mathrm{eV}$

\begin{tabular}{llll}
\hline Site & Position & $\mathrm{Pt}_{116}-\mathrm{O}$ & $\mathrm{Pt}_{116}-\mathrm{O}_{2}$ \\
\hline 1 & Top & $-{ }^{a}$ & $--^{b}$ \\
2 & Top & -4.26 & -0.66 \\
3 & Top & -3.92 & $--^{c}$ \\
4 & Top & -3.58 & $-{ }^{d}$ \\
5 & Bridge & $-{ }^{f}$ & -0.83 \\
6 & Bridge & $-{ }^{a}$ & -0.93 \\
7 & Bridge & -4.92 & -1.17 \\
8 & Bridge & -5.02 & -1.87 \\
9 & Bridge & -4.76 & -1.68 \\
10 & Bridge & -4.66 & -1.34 \\
11 & hcp & -4.65 & -0.99 \\
12 & fcc & -4.42 & -0.88 \\
13 & hcp & -4.79 & -0.68 \\
14 & fcc & $-{ }^{c}$ & -1.07 \\
15 & 4-Fold hollow & & $-{ }^{d}$
\end{tabular}

${ }^{a}$ Adsorbate migration to position $14 .{ }^{b}$ Adsorbate migration to position 13 . ${ }^{c}$ Adsorbate migration to position 9. ${ }^{d}$ Adsorbate migration to positions 9 and $10 .{ }^{e}$ Adsorbate migration to position $12 .{ }^{f}$ Adsorbate migration to position 11.

when compared to the hollow positions. This indicates that, as the particle size increases, there is a move away from the hollow sites directed towards the edge-bridge sites. The exact edgebridge position also varies as seen when comparing $\mathrm{Pt}_{79}$, where the edge-bridge position between two (111) facets is favourable, to $\mathrm{Pt}_{116}$, where the edge-bridge site between the (111) and (100) facets is favourable.

$\mathrm{O}_{2}$ is preferentially adsorbed at position 8, the edge-bridge site between the two (111) facets. This is in agreement with results for $\mathrm{Pt}_{79}$, although it is found that the edge-bridge site between the (111) and (100) facets is most favourable for $\mathrm{Pt}_{38}$. $\mathrm{O}_{2}$ at the edge-bridge site is found to be $\geq 0.17 \mathrm{eV}$ lower in energy than at hollow sites on $\mathrm{Pt}_{38}$, suggesting more site preference for $\mathrm{O}_{2}$ binding when compared with atomic oxygen. This is also seen for the 79- and 116-atom species, where the preference for binding to edge-bridge sites over hollow sites is $\geq 0.70$ and $\geq 0.80 \mathrm{eV}$, respectively. This also manifests size dependence, where the energy difference between the edgebridge and hollow sites increases as particle size increases.

$\mathrm{O}_{2}$ dissociation is investigated for sites on both the (111) and (100) facets. For $\mathrm{Pt}_{116}$, bridge sites 8, 9 and 10 have been investigated as well as hollow sites 11,12 and $14 . \mathrm{O}_{2}$ dissociation proceeds from an initial site and terminates at the central hollow position on the (111) facet (site 13). Any further migration of the atomic oxygen to other positions on the particle would lead to additional small barriers associated with moving between different sites but would have little importance. The central hollow site of the (111) facet was also investigated, although found to be unfavourable for $\mathrm{O}_{2}$ dissociation, which is likely due to the small (in magnitude) $\mathrm{O}_{2}$ binding energy of $-0.68 \mathrm{eV}$.

\subsection{Barriers for $\mathrm{O}_{2}$ dissociation}

Representations of the minimised geometries for the initial, transition and final states (IS, TS and FS, respectively) can be found in the ESI, $\dagger$ Fig. S1-S6. For comparative purposes, the minimised geometries for the initial, transition and final states of the extended Pt(111) surface can be found in the ESI, $\dagger$ Fig. S7. A greater range of facet sites were studied for $\mathrm{Pt}_{116}$, these included similar (111) hollow sites to those investigated previously (positions 11, 12, 13 and 14) but also a greater range of bridge sites (positions 5, 6, 7, 8, 9, and 10). As with $\mathrm{Pt}_{79}$, the (111) hollow site at the centre of the (111) facet, position 13, is found to exhibit weak Pt-O binding. This explains why the attempted $\mathrm{O}_{2}$ dissociation studies were not successful, where the $\mathrm{O}_{2}$ molecule favours migration away from the initial facet position, towards a more favourable one, instead of dissociating.

Relatively low binding energies are found for bridge positions 5 , 6 and $7,-0.83$ to $-1.17 \mathrm{eV}$. Instead of $\mathrm{O}_{2}$ dissociation barriers being located for these positions, small barriers, $\sim 0.1 \mathrm{eV}$ are found for $\mathrm{O}_{2}$ migration to a preferential site, e.g. an edge-hollow site. This suggests that, where possible, $\mathrm{O}_{2}$ migrates to favourable binding sites prior to dissociation. Details for the positions at which dissociation barriers were located are given in Table 2.

For $\mathrm{Pt}_{116}$ there are very low dissociation barriers for hollow positions 11, 12 and 14, at 0.08, 0.07 and $0.14 \mathrm{eV}$, respectively. Although the barrier at position 14 is slightly higher than those for 11 and 12, it is significantly lower than that of the bulk $\left(\sim 0.40 \mathrm{eV}^{46}\right)$. Furthermore, it is comparable with that of position 9 on $\mathrm{Pt}_{79}$, which was found to be $0.21 \mathrm{eV}$. Whilst position 9 on $\mathrm{Pt}_{79}$ is the hcp hollow site adjacent to a (100) facet, position 14 on $\mathrm{Pt}_{116}$ is the fcc hollow site adjacent to another (111) facet site. There are few similarities between the two sites that could

Table 2 Particle $\left(\Delta E\left(\mathrm{Pt}_{116}\right)\right)$ and oxygen $\left(\Delta E\left(\mathrm{O}_{2}\right)\right)$ distortion energies, root mean squared displacements (RMSD) of the particle, interaction energies $\left(E_{\text {int }}\right)$, binding energies $\left(E_{\mathrm{b}}\right)$ of $\mathrm{O}_{2}$ and barrier of $\mathrm{O}_{2}$ dissociation $\left(\Delta E^{\neq}\right)$. All energies are given in eV and RMSD in $\AA$

\begin{tabular}{|c|c|c|c|c|c|c|}
\hline & $\Delta E\left(\mathrm{Pt}_{116}\right)$ & RMSD & $\Delta E\left(\mathrm{O}_{2}\right)$ & $E_{\text {int }}$ & $E_{\mathrm{b}}$ & $\Delta E^{\neq}$ \\
\hline \multicolumn{7}{|l|}{ Initial state } \\
\hline $\mathrm{Pt}_{116}-8$ & 0.10 & 0.04 & 0.70 & -2.67 & 1.87 & \\
\hline $\mathrm{Pt}_{116^{-9}}$ & 0.38 & 0.14 & 0.56 & -2.63 & 1.68 & \\
\hline $\mathrm{Pt}_{116}-10$ & 0.34 & 0.11 & 0.51 & -2.19 & -1.34 & \\
\hline $\mathrm{Pt}_{116}-11$ & 0.20 & 0.07 & 0.84 & -2.03 & -0.99 & \\
\hline $\mathrm{Pt}_{116}-12$ & 0.65 & 0.16 & 0.91 & -2.43 & -0.88 & \\
\hline $\mathrm{Pt}_{116}-14$ & 0.23 & 0.07 & 0.91 & -2.21 & -1.07 & \\
\hline Pt(111) slab & $0.17^{a}$ & $0.03^{b}$ & 0.64 & -1.67 & -0.86 & \\
\hline \multicolumn{7}{|c|}{ Transition state } \\
\hline $\mathrm{Pt}_{116^{-8}}$ & 0.21 & 0.09 & 5.15 & -6.91 & -1.56 & 0.32 \\
\hline $\mathrm{Pt}_{116}-9$ & 0.47 & 0.16 & 5.58 & -7.33 & -1.27 & 0.41 \\
\hline $\mathrm{Pt}_{116}-10$ & 0.44 & 0.14 & 5.84 & -6.92 & -0.64 & 0.69 \\
\hline $\mathrm{Pt}_{116}-11$ & 0.84 & 0.21 & 2.35 & -4.11 & -0.91 & 0.08 \\
\hline $\mathrm{Pt}_{116}-12$ & 0.72 & 0.17 & 2.81 & -4.33 & -0.80 & 0.07 \\
\hline $\mathrm{Pt}_{116^{-14}}$ & 0.41 & 0.10 & 3.35 & -4.69 & -0.93 & 0.14 \\
\hline $\operatorname{Pt}(111)$ slab & $0.25^{a}$ & $0.05^{b}$ & 5.72 & -6.41 & -0.43 & 0.45 \\
\hline \multicolumn{7}{|l|}{ Final state } \\
\hline $\mathrm{Pt}_{116^{-8}}$ & 0.53 & 0.09 & 9.65 & -13.41 & -3.23 & \\
\hline $\mathrm{Pt}_{116^{-9}}$ & 0.52 & 0.12 & 9.31 & -12.77 & -2.94 & \\
\hline $\mathrm{Pt}_{116}-10$ & 0.65 & 0.13 & 9.35 & -12.50 & -2.50 & \\
\hline $\mathrm{Pt}_{116^{-11}}$ & 1.03 & 0.16 & 9.22 & -12.56 & -2.30 & \\
\hline $\mathrm{Pt}_{116}-12$ & 1.37 & 0.21 & 8.20 & -11.36 & -1.78 & \\
\hline $\mathrm{Pt}_{116}-14$ & 0.72 & 0.13 & 9.49 & -12.41 & -2.19 & \\
\hline $\operatorname{Pt}(111)$ slab & $0.39^{a}$ & $0.05^{b}$ & 6.34 & -9.12 & -2.39 & \\
\hline
\end{tabular}


lead to this slightly higher dissociation barrier aside from the fact that they are both adjacent to the "short" edge of the (111) facet. On $\mathrm{Pt}_{79}$, there are two hollow positions adjacent to (111) facets, and only one adjacent to the (100) facets. For $\mathrm{Pt}_{116}$ there are two hollow sites adjacent to the (100) facets, but only one adjacent to the (111) facets. It is therefore likely that the facets on the "short" edge are slightly more difficult to distort, with the bridge bond between facets being more ridgid. However, as the central atoms of the (111) facet are more flexible, favourable distortions towards the centre of the facet are still possible, leading to these slightly higher dissociation barriers compared to other hollow positions on the (111) facet, but still lower than the Pt slab. Dissociation barriers for the $\mathrm{Pt}_{38}$ and $\mathrm{Pt}_{79}$ particles are presented in Table 3, along with those for the $\mathrm{Pt}_{116}$ particles.

\subsection{Distortion energies}

Studies of the IS reveal few trends in the distortion energies of $\mathrm{Pt}_{116}$ upon adsorption of $\mathrm{O}_{2}$. Compared to other positions, there is a small distortion energy for $\mathrm{Pt}_{116}$ when $\mathrm{O}_{2}$ is adsorbed on position 8 , the edge-bridge between two (111) facets. When compared to the $\mathrm{Pt}$ slab, sites 8,11 and 14 have similar distortion energies ( $\leq 0.07 \mathrm{eV}$ difference). For $\mathrm{Pt}_{38}$, none of the nanoparticle distortion energies are close to that of the slab ( $\geq 0.25 \mathrm{eV}$ difference). For $\mathrm{Pt}_{79}$, site 9 is $0.04 \mathrm{eV}$ lower in energy than the slab, however other sites are $\geq 0.14 \mathrm{eV}$ higher in energy. This shows a trend towards infinite surface like behaviour with increasing particle size. The greatest distortion energy on $\mathrm{Pt}_{116}$ is found for position 12, $0.65 \mathrm{eV}$, although the distortion energies for the various sites are generally in line with those for the 38- and 79-atom particles of $\leq 0.50 \mathrm{eV}$.

At the TS, position 8 once again has the lowest distortion energy associated with it. The particle distortion energy for site 8 is similar to that of the slab, 0.21 and $0.25 \mathrm{eV}$, respectively. However, all other sites have significantly higher distortion energies associated with them, 0.44-0.84 eV. The greatest distortion energies are found for positions 11 and 12 which are indicative of low $\mathrm{O}_{2}$ dissociation barriers. The particle distortion energy for site 14 is similar to those of positions 9 and 10 , although a significantly lower $\mathrm{O}_{2}$ dissociation barrier is found for 14 compared to 9 and 10 . The dissociation barrier for position 14 lies between the other low barrier (111) hollow sites

Table 3 Dissociation barriers $\Delta E^{\neq}(\mathrm{eV})$ for the 38, 79 and 116 atom particles at the TS. The value for the $\mathrm{Pt}(111)$ slab has also been calculated for comparison. ${ }^{a}$ Data presented from the previous study for comparative purposes ${ }^{46}$

\begin{tabular}{|c|c|c|c|c|c|}
\hline \multicolumn{2}{|c|}{$\mathrm{Pt}_{38}$} & \multicolumn{2}{|l|}{$\mathrm{Pt}_{79}$} & \multicolumn{2}{|c|}{$\mathrm{Pt}_{116}$} \\
\hline Site & $\Delta E^{f}$ & Site & $\Delta E^{f}$ & Site & $\Delta E^{f}$ \\
\hline 5 & 0.32 & 8 & 0.34 & 8 & 0.32 \\
\hline 6 & 0.04 & 9 & 0.21 & 9 & 0.41 \\
\hline \multirow[t]{4}{*}{7} & 0.00 & 10 & 0.00 & 10 & 0.69 \\
\hline & & 11 & 0.05 & 11 & 0.08 \\
\hline & & & & 12 & 0.07 \\
\hline & & & & 14 & 0.14 \\
\hline
\end{tabular}

${ }^{a} \Delta E^{f}$ for the $\operatorname{Pt}(111)$ surface is $0.45 \mathrm{eV}$. and the high barrier bridge sites. There is a slight increase in the distortion energies when comparing the IS and TS structures, where the TS ones tend to have greater distortion energies. Furthermore, the distortion energies at the TS for $\mathrm{Pt}_{116}$ tend to be greater than those for the $\mathrm{Pt}_{38}$ and $\mathrm{Pt}_{79}$ TS structures.

At the FS, the $\mathrm{Pt}_{116}$ distortion energies are all greater than for the Pt slab. Furthermore, positions 8 and 9 have comparable distortion energies, both $\sim 0.10 \mathrm{eV}$ higher in energy than the slab model. The $\mathrm{Pt}_{116}$ distortion energies for positions 8 and 9 are lower than other sites as adsorption on the bridge positions typically leads to less distortion. Position 10 has a larger distortion energy compared to the other bridge positions. In this case, $\mathrm{O}_{2}$ dissociation occurs over the central atom of the (100) facet. This leads to similar distortions to the (111) facet, with the central atom being pulled out of the plane. However, for the (100) facet, this distortion does not lead to the very low dissociation barriers observed for the (111) facet. Instead, a high barrier of $0.69 \mathrm{eV}$ is calculated. Position 14 has a higher distortion energy than the bridge sites, although this is lower than the distortion energy for the other hollow sites. As discussed previously, there is less distortion of the "short" facet edge, leading to a reduced distortion energy compared to positions 11 and 12 .

RMSD is calculated for all particles, however only the atoms to which $\mathrm{O}_{2}$ is bound as well as nearest neighbour Pt atoms are accounted for. There are generally greater displacement associated with the TS and FS. This is expected as the oxygen binds more strongly to the Pt surface as the system progresses from the IS to the FS. There are fewer trends when comparing the various sites being studied. Generally, there is a large RMSD associated with sites 11 and 12 (particularly at the TS), which exhibit larger distortion energies and lower $\mathrm{O}_{2}$ dissociation barriers compared to other sites.

At the IS, the oxygen distortion energy shows that there is greater distortion of $\mathrm{O}_{2}$ on the hollow sites, positions 11, 12 and 14. This means that at the positions for which there are very low dissociation barriers, there is greater distortion, away from the gas phase structure, of $\mathrm{O}_{2}$. Conversely at the TS, this trend inverts, where higher $\mathrm{O}_{2}$ distortion barriers are found for the bridge sites, 8, 9 and 10. This shows that at the TS, there is less distortion of the $\mathrm{O}_{2}$ on the hollow sites.

\subsection{Interaction energies}

$E_{\text {int }}$ gives an idea of the strength of the $\mathrm{O}_{2}$ bond to the surface. From the IS studies it follows that there is little difference between the interaction energies for the various sites. This trend changes at the TS, where greater interaction energies are found for the bridge sites than for the hollow sites. Thus, at the bridge sites, where there is typically less distortion of $\mathrm{Pt}_{116}$ and greater distortion of $\mathrm{O}_{2}$, there is a stronger Pt-O interaction. This is the opposite trend to that of the hollow sites, where there is greater distortion of $\mathrm{Pt}_{116}$ and less of $\mathrm{O}_{2}$. Similar trends to this are found for the binding energy, although in this case the trends are not as obvious because $E_{\mathrm{b}}$ includes the energetic contributions of distortions compared to the gas phase structures. 


\subsection{Geometric analysis}

Calculated geometric parameters are listed in Table 4. At the IS, there is generally little difference in the $\mathrm{O}-\mathrm{O}$ bond length for the various sites. However, at the TS, shorter $\mathrm{O}-\mathrm{O}$ distances are found on the hollow sites, compared to the bridge sites. On the hollow sites, the O-O distances are on average only $0.25 \AA$ longer at the TS compared to the IS. This compares to an $0.84 \AA$ average increase in the $\mathrm{O}-\mathrm{O}$ distances from the TS to the IS on the bridge sites. This trend is in good agreement with results of the previous studies on $\mathrm{Pt}_{38}$ and $\mathrm{Pt}_{79}$ which exhibit early transition states located on the (111) facet where $\mathrm{O}_{2}$ dissociation results in very small energetic barriers. As the $\mathrm{Pt}_{116}$ system progresses from the IS to the TS, the $\mathrm{Pt}-\mathrm{O}$ bond lengths are shortest at the TS. Pt-O bond lengths are increased when comparing the FS to the TS, although those at the FS are generally still shorter than at the IS. This is due to the increased binding energies observed as the system progresses from the IS to the FS, leading to the reduction in the Pt-O bond length. Longer Pt-O bonds are observed when $\mathrm{O}_{2}$ is adsorbed on the hollow sites, compared to the bridge sites.

When inspecting Pt-Pt bond lengths, several trends become apparent. On the bridge sites, the Pt-Pt TS bond is elongated compared to the IS, whilst the FS bond is generally shorter than those for the IS and TS. The TS will have the longest Pt-Pt bond because of the stronger Pt-O interactions when compared to the IS. The Pt-Pt bond lengths are then generally reduced for the FS because, whilst the $\mathrm{Pt}-\mathrm{O}$ binding is stronger, it is only a single $\mathrm{O}$ atom occupying the site. The trends are less well defined for the hollow sites, although in this case, there are a greater number of bonds that can be distorted. This can lead to smaller distortion of a certain bond type, compared to the bridge sites where $\leq 2$ bonds are being distorted. $\mathrm{Pt}-\mathrm{X}^{\text {centre }}$ values are calculated for all hollow sites. In all cases, there is a greater distortion of the central (111) facet atom at the TS and FS when compared to the IS. This shows that as the Pt-O interaction energy increases, the (111) facet distorts more.

\subsection{Bader charge analysis}

Results from the Bader charge analysis are given in Table 5. Charge density difference plots are shown in the ESI, $\dagger$ Fig. S8-S14.
Consistent with previous studies, ${ }^{41,42,46,60}$ there is significant charge transfer from the Pt, which becomes positively charged, to the $\mathrm{O}$, which becomes negatively charged. At the IS it is found that there is less charge transfer from Pt for site 8 compared to sites 9 and 10. Instead, the charge state of site 8 is comparable to the hollow sites, which are slightly less positively charged. When studying $\mathrm{O}_{2}$ charge, it is found that there is a greater charge compared to the $\mathrm{O}$ associated with the hollow sites. For the hollow sites, charge transfer is investigated for the three Pt atoms directly bonded to $\mathrm{O}_{2}$, instead of the two atoms of the bridge sites. This means that, although there is typically less charge transfer from one Pt atom, as there are more Pt atoms interacting with $\mathrm{O}$, the average charge on the $\mathrm{O}$ is greater.

The charge transfer at the TS is greater than that at the IS. As the $\mathrm{O}_{2}$ dissociates, the interaction energy increases, coupled with a greater transfer of charge from the Pt to the O. At the TS, there is greater charge transfer at the bridge sites which is due to the coordination number of the $\mathrm{O}$, where each $\mathrm{O}$ atom is bonded to a single Pt atom. For the hollow sites the charge transfer is spread over three Pt atoms, which results in the smaller transfer of charge. A similar trend is observed for the FS, where once again, greater charge transfer is found than at the IS or TS. Each $\mathrm{O}$ atom is now strongly bonded to the Pt surface and able to extract a greater charge than the molecular $\mathrm{O}_{2}$. At the FS, there is less difference in charge transfer between the bridge and hollow sites. This is because the transferred charge is spread over a greater number of atoms at each site.

Table 5 Average charges (|e|) of the initial (IS), transition (TS) and final (FS) state structures of $\mathrm{O}_{2}$ dissociation on $\mathrm{Pt}_{116}$

\begin{tabular}{|c|c|c|c|c|c|c|}
\hline \multirow[b]{2}{*}{ Site } & \multicolumn{3}{|c|}{ Pt charges $^{a}$} & \multicolumn{3}{|c|}{ O charges } \\
\hline & IS & TS & FS & IS & TS & FS \\
\hline $\mathrm{Pt}_{116}-8$ & 0.19 & 0.46 & 0.32 & -0.33 & -0.54 & -0.75 \\
\hline $\mathrm{Pt}_{116}-9$ & 0.29 & 0.51 & 0.44 & -0.33 & -0.58 & -0.72 \\
\hline $\mathrm{Pt}_{116}-10$ & 0.28 & 0.49 & 0.45 & -0.31 & -0.59 & -0.73 \\
\hline $\mathrm{Pt}_{116}-11$ & 0.20 & 0.28 & 0.31 & -0.36 & -0.45 & -0.77 \\
\hline $\mathrm{Pt}_{116}-12$ & 0.24 & 0.31 & 0.39 & -0.38 & -0.48 & -0.75 \\
\hline $\mathrm{Pt}_{116}-14$ & 0.19 & 0.28 & 0.30 & -0.37 & -0.49 & -0.76 \\
\hline
\end{tabular}

${ }^{a}$ Pt atoms interacting directly with $\mathrm{O}$ atoms.

Table 4 Data for oxygen-oxygen distances $(\mathrm{O}-\mathrm{O})$, average platinum-oxygen bond lengths (Pt-O), average platinum-platinum bond lengths (Pt-Pt) for specific bonds defined in Fig. 1 and distances from the octahedral core to the central (111) atom in the $y$-direction (Pt-Xcentre). Average Pt-Pt bond lengths are calculated for those atoms directly below the adsorbed $\mathrm{O}_{2}$ molecule. Bond lengths are given in $\AA$. The type of the bond according to Fig. 1 is shown as a superscript

\begin{tabular}{|c|c|c|c|c|c|c|c|c|c|c|c|c|}
\hline \multirow[b]{2}{*}{ Site } & \multicolumn{3}{|l|}{$\mathrm{O}-\mathrm{O}$} & \multicolumn{3}{|l|}{$\mathrm{Pt}-\mathrm{O}$} & \multicolumn{3}{|l|}{$\mathrm{Pt}-\mathrm{Pt}$} & \multicolumn{3}{|c|}{$\underline{\mathrm{Pt}}-\mathrm{X}^{\text {centre }}$} \\
\hline & IS & TS & FS & IS & TS & FS & IS & TS & FS & IS & $\mathrm{TS}$ & FS \\
\hline $\mathrm{Pt}_{116^{-8}}$ & 1.40 & 2.00 & 5.58 & 1.97 & 1.82 & 1.94 & $2.78^{1}$ & $3.03^{2}$ & $2.98^{2}$ & - & - & - \\
\hline $\mathrm{Pt}_{116}-9$ & 1.38 & 2.21 & 3.24 & 1.96 & 1.80 & 1.94 & $3.16^{2}$ & $3.22^{2}$ & $2.91^{2}$ & - & - & - \\
\hline $\mathrm{Pt}_{116}-10$ & 1.38 & 2.47 & 3.29 & 1.98 & 1.81 & 1.95 & $3.10^{3}$ & $3.14^{3}$ & $2.93^{3}$ & - & - & - \\
\hline $\mathrm{Pt}_{116^{-11}}$ & 1.42 & 1.62 & 3.13 & 2.12 & 2.01 & 2.05 & $\begin{array}{l}2.78^{2} \\
2.76^{4} \\
2.87^{5}\end{array}$ & $\begin{array}{l}2.78^{2} \\
2.31^{4} \\
3.19^{5}\end{array}$ & $\begin{array}{l}2.86^{2} \\
3.12^{4} \\
2.88^{5} \\
3.00^{6}\end{array}$ & 4.03 & 4.65 & 4.48 \\
\hline $\mathrm{Pt}_{116}-12$ & 1.43 & 1.67 & 2.52 & 2.07 & 1.99 & 2.07 & $\begin{array}{l}3.33^{4} \\
2.92^{6}\end{array}$ & $\begin{array}{l}3.35^{4} \\
2.93^{6}\end{array}$ & $\begin{array}{l}3.21^{4} \\
3.03^{6}\end{array}$ & 3.99 & 4.04 & 4.41 \\
\hline $\mathrm{Pt}_{116^{-14}}$ & 1.43 & 1.74 & 3.62 & 2.12 & 2.00 & 2.05 & $\begin{array}{l}2.79^{1} \\
2.93^{5}\end{array}$ & $\begin{array}{l}2.83^{1} \\
3.06^{5}\end{array}$ & $\begin{array}{l}2.86^{1} \\
2.87^{5} \\
2.96^{6}\end{array}$ & 4.03 & 4.12 & 4.11 \\
\hline
\end{tabular}




\section{Conclusions}

We have presented a comprehensive study of size effects on $\mathrm{O}_{2}$ dissociation of $\mathrm{Pt}$ particles considering the $\mathrm{Pt}_{116}$ particle and comparing with the previously obtained results for smaller Pt particles. Most of the trends observed in the previous studies on $\mathrm{Pt}_{38}$ and $\mathrm{Pt}_{79}$ species are also seen for the larger $\mathrm{Pt}_{116}$. It is seen that the small $\mathrm{O}_{2}$ dissociation barriers, first calculated on $\mathrm{Pt}_{38}$, were not due to the small particle size. For the $\mathrm{Pt}_{116}$ model much lower dissociation barriers are obtained on the (111) facet hollow sites at the edge between (111) and (100) facets, $0.07 \mathrm{eV}$, when compared to the Pt(111) slab, $0.45 \mathrm{eV}$. A slightly higher dissociation barrier, $0.14 \mathrm{eV}$, is found for the other hollow site located close to the edge between two neighbouring (111) facets, although this is significantly lower than for the Pt slab, or other bridge sites on the $\mathrm{Pt}_{116}$ particle.

For $\mathrm{Pt}_{116}$, other sites were also investigated: these included bridge sites on the (111) and (100) facets as well as the edge bridge sites between neighbouring (111) facets and between (111) and (100) facets. When studying the bridge sites on the (111) facet, it was found that the $\mathrm{O}_{2}$ would, instead of dissociating, overcome low barriers to migrate to hollow sites. Both edge bridge sites exhibited similar characteristics. On the other hand, it was found that there was a higher dissociation barrier for the bridge site at (100) facets, when compared to the edge bridge sites. This demonstrates that $\mathrm{O}_{2}$ dissociation will occur preferentially on (111) facets near the nanoparticle edges and the advantages of particles with greater numbers of (111) facet sites compared to (100) facet sites.

\section{Acknowledgements}

This research was funded through the RCUK doctoral training centre in hydrogen, fuel cells and their applications (EP/ G037116/1). Financial support was also provided by the EU (FP7-NMP.2012.1.1-1 project ChipCAT, Ref. No. 310191), the Generalitat de Catalunya (2014SGR97, XRQTC) and the Spanish MICINN (CTQ2012-34969). The authors thank COST Actions MP0903 and CM1104. Much of the initial work was performed under the HPC-EUROPA2 project (project number: 228398) with the support of the European Commission - Capacities Area - Research Infrastructures. The computations described in this paper were performed in part using The University of Birmingham's BlueBEAR HPC service, which was purchased through HEFCE SRIF-3 funds, and via our membership of the UK's HPC Materials Chemistry Consortium, which is funded by EPSRC (EP/F067496). This work made use of the facilities of HECToR, the UK's national high-performance computing service, which is provided by UoE HPCx Ltd at the University of Edinburgh, Cray Inc and NAG Ltd, and funded by the Office of Science and Technology through EPSRC's High End Computing Programme. H. A. A. thanks the Spanish Ministerio de Educación for a post-doctoral grant (SB2010-0172), Bulgarian Science Fund (grant DCVP 02/1) and FP7 project BeyondEverest for support.

\section{References}

1 A. Chandan, M. Hattenberger, A. El-kharouf, S. Du, A. Dhir, V. Self, B. G. Pollet, A. Ingram and W. Bujalski, J. Power Sources, 2013, 231, 264-278.

2 S. Guo, S. Zhang and S. Sun, Angew. Chem., Int. Ed., 2013, 52, 8526-8544.

3 Z. Liu, L. Ma, J. Zhang, K. Hongsirikarn and J. G. Goodwin, Catal. Rev., 2013, 55, 255-288.

4 L. Su, W. Jia, C.-M. Li and Y. Lei, ChemSusChem, 2014, 7, 361-378.

5 J. Greeley, I. E. L. Stephens, A. S. Bondarenko, T. P. Johansson, H. A. Hansen, T. F. Jaramillo, J. Rossmeisl, I. Chorkendorff and J. K. Nørskov, Nat. Chem., 2009, 1, 552-556.

6 V. Viswanathan, H. A. Hansen, J. Rossmeisl and J. K. Nørskov, ACS Catal., 2012, 2, 1654-1660.

7 T. P. Johansson, E. T. Ulrikkeholm, P. Hernandez-Fernandez, P. Malacrida, H. A. Hansen, A. S. Bandarenka, J. K. Nørskov, J. Rossmeisl, I. E. L. Stephens and I. Chorkendorff, Top. Catal., 2013, 57, 245-254.

8 A. B. Anderson and T. V. Albu, J. Electrochem. Soc., 2000, 147, 4229.

9 R. Jinnouchi, Microscale Thermophys. Eng., 2003, 7, 15-31.

10 J. K. Nørskov, J. Rossmeisl, A. Logadottir, L. Lindqvist, J. R. Kitchin, T. Bligaard and H. Jónsson, J. Phys. Chem. B, 2004, 108, 17886-17892.

11 J. A. Keith, G. Jerkiewicz and T. Jacob, ChemPhysChem, 2010, 11, 2779-2794.

12 Y. Sha, T. H. Yu, B. V. Merinov, P. Shirvanian and W. A. Goddard, J. Phys. Chem. Lett., 2011, 2, 572-576.

13 M. T. M. Koper, T. E. Shubina and R. A. van Santen, J. Phys. Chem. B, 2002, 106, 686-692.

14 J. Seminario, L. Agapito, L. Yan and P. Balbuena, Chem. Phys. Lett., 2005, 410, 275-281.

15 B. Han, C. Miranda and G. Ceder, Phys. Rev. B: Condens. Matter Mater. Phys., 2008, 77, 075410.

16 Q. Ge, P. Hu, D. A. King, M.-H. Lee, J. A. White and M. C. Payne, J. Chem. Phys., 1997, 106, 1210.

17 K. Bleakley and P. Hu, J. Am. Chem. Soc., 1999, 121, 7644-7652. 18 H. Tang, A. Van der Ven and B. Trout, Phys. Rev. B: Condens. Matter Mater. Phys., 2004, 70, 045420.

19 X. Lin, N. J. Ramer, A. M. Rappe, K. C. Hass, W. F. Schneider and B. L. Trout, J. Phys. Chem. B, 2001, 105, 7739-7747.

20 T. Li and P. B. Balbuena, J. Phys. Chem. B, 2001, 105, 9943-9952.

21 T. Jacob, R. P. Muller and W. A. Goddard, J. Phys. Chem. B, 2003, 107, 9465-9476.

22 G. N. Vayssilov, Y. Lykhach, A. Migani, T. Staudt, G. P. Petrova, N. Tsud, T. Skála, A. Bruix, F. Illas, K. C. Prince, V. Matoln, K. M. Neyman and J. Libuda, Nat. Mater., 2011, 10, 310-315.

23 T. Q. Nguyen, M. C. S. Escaño, H. Nakanishi, H. Kasai, H. Maekawa, K. Osumi and K. Sato, Appl. Surf. Sci., 2014, 288, 244-250.

24 U. Heiz, A. Sanchez, S. Abbet and W.-D. Schneider, J. Am. Chem. Soc., 1999, 121, 3214-3217.

25 R. L. Johnston, Atomic \& Molecular Clusters, Taylor \& Francis, London, 2002, p. 208. 
26 S. M. Kozlov and K. M. Neyman, Top. Catal., 2013, 56, 867-873.

27 H. A. Aleksandrov, F. Viñes, W. Ludwig, S. Schauermann and K. M. Neyman, Chem. - Eur. J., 2013, 19, 1335-1345.

28 F. Viñes, Y. Lykhach, T. Staudt, M. P. A. Lorenz, C. Papp, H.-P. Steinrück, J. Libuda, K. M. Neyman and A. Görling, Chem. - Eur. J., 2010, 16, 6530-6539.

29 R. B. Getman, Y. Xu and W. F. Schneider, J. Phys. Chem. C, 2008, 112, 9559-9572.

30 A. Smeltz, R. Getman, W. Schneider and F. Ribeiro, Catal. Today, 2008, 136, 84-92.

31 R. Getman, W. Schneider, A. Smeltz, W. Delgass and F. Ribeiro, Phys. Rev. Lett., 2009, 102, 076101.

32 W. Chen, D. Schmidt, W. F. Schneider and C. Wolverton, J. Phys. Chem. C, 2011, 115, 17915-17924.

33 J. M. Bray and W. F. Schneider, Langmuir, 2011, 27, 8177-8186.

34 Q. Fu, J. Yang and Y. Luo, J. Phys. Chem. C, 2011, 115, 6864-6869.

35 M. Stamatakis and D. G. Vlachos, ACS Catal., 2012, 2, 2648-2663.

36 D. J. Schmidt, W. Chen, C. Wolverton and W. F. Schneider, J. Chem. Theory Comput., 2012, 8, 264-273.

37 Y. Xu, W. A. Shelton and W. F. Schneider, J. Phys. Chem. A, 2006, 110, 5839-5846.

38 D.-H. Lim and J. Wilcox, J. Phys. Chem. C, 2011, 115, 22742-22747.

39 M. Kettner, W. B. Schneider and A. A. Auer, J. Phys. Chem. C, 2012, 116, 15432-15438.

40 D. Cheng and W. Wang, Nanoscale, 2012, 4, 2408-2415.

41 P. C. Jennings, B. G. Pollet and R. L. Johnston, Phys. Chem. Chem. Phys., 2012, 14, 3134-3139.

42 P. C. Jennings, B. G. Pollet and R. L. Johnston, J. Phys. Chem. C, 2012, 116, 15241-15250.

43 B. Hammer and J. Nørskov, Surf. Sci., 1995, 343, 211-220.

44 V. R. Stamenkovic, B. S. Mun, M. Arenz, K. J. J. Mayrhofer, C. A. Lucas, G. Wang, P. N. Ross and N. M. Markovic, Nat. Mater., 2007, 6, 241-247.
45 S. J. Yoo, S. J. Hwang, J.-G. Lee, S.-C. Lee, T.-H. Lim, Y.-E. Sung, A. Wieckowski and S.-K. Kim, Energy Environ. Sci., 2012, 5, 7521-7525.

46 P. C. Jennings, H. A. Aleksandrov, K. M. Neyman and R. L. Johnston, Nanoscale, 2014, 6, 1153-1165.

47 A. H. Larsen, J. Kleis, K. S. Thygesen, J. K. Nørskov and K. W. Jacobsen, Phys. Rev. B: Condens. Matter Mater. Phys., 2011, 84, 245429.

48 G. Kresse and J. Hafner, Phys. Rev. B: Condens. Matter Mater. Phys., 1993, 47, 558-561.

49 G. Kresse and J. Hafner, Phys. Rev. B: Condens. Matter Mater. Phys., 1994, 49, 14251-14269.

50 G. Kresse, Phys. Rev. B: Condens. Matter Mater. Phys., 1996, 54, 11169-11186.

51 G. Kresse and J. Furthmüller, Comput. Mater. Sci., 1996, 6, 15-50.

52 P. E. Blöchl, Phys. Rev. B: Condens. Matter Mater. Phys., 1994, 50, 17953-17979.

53 G. Kresse, Phys. Rev. B: Condens. Matter Mater. Phys., 1999, 59, 1758-1775.

54 J. P. Perdew, K. A. Jackson, M. R. Pederson, D. J. Singh and C. Fiolhais, Phys. Rev. B: Condens. Matter Mater. Phys., 1992, 46, 6671-6687.

55 J. Perdew, J. Chevary, S. Vosko, K. Jackson, M. Pederson, D. Singh and C. Fiolhais, Phys. Rev. B: Condens. Matter Mater. Phys., 1993, 48, 4978.

56 D. Sheppard, P. Xiao, W. Chemelewski, D. D. Johnson and G. Henkelman, J. Chem. Phys., 2012, 136, 074103.

57 J. Kästner and P. Sherwood, J. Chem. Phys., 2008, 128, 014106.

58 R. F. W. Bader, Atoms in Molecules: a Quantum Theory, Oxford University Press, New York, 1994.

59 W. Tang, E. Sanville and G. Henkelman, J. Phys.: Condens. Matter, 2009, 21, 084204.

60 P. C. Jennings and R. L. Johnston, Comput. Theor. Chem., 2013, 1021, 91-100. 